\title{
TOWARDS SYSTEMS INTEGRATION THEORY IN MEGAPROJECTS: A SYSTEM-OF-SYSTEMS FRAMEWORK
}

\author{
Jin $\mathrm{Zhu}^{1}$, Ali Mostafavi ${ }^{2}$, and Jennifer Whyte ${ }^{3}$
}

\begin{abstract}
Megaprojects are large-scale, complex projects composed of various interconnected constituents. Recent research suggests that effective systems integration is one of the major challenges affecting the success of their delivery and that we hence need new taxonomies to characterise and evaluate integration in this context. This paper draws on and contributes to a growing literature on systems integration in megaprojects by proposing a formalized framework for characterization and evaluation of integration between constituents of megaprojects. In this study, megaprojects are conceptualized as systems-of-systems (SoSs). Based on this conceptualization, a SoS framework towards a systems integration theory in megaproject SoSs is proposed. The proposed framework includes two aspects. The first articulates the different dimensions of systems integration. It identifies the types of integration; focuses of integration; integration at different levels; and integration in different phases in megaproject SoSs. The second aspect is related to the indicators of systems integration. Seven indicators are discussed for evaluating the degree of integration in megaprojects. The proposed SoS framework provides a theoretical basis for future research on systems integration in megaprojects and can be used as a guide for developing corresponding empirical studies.
\end{abstract}

Keywords: megaprojects, systems integration, system-of-systems.

\section{INTRODUCTION}

Megaprojects are large-scale, complex ventures that typically cost US\$1 billion or more, take many years to develop and build, involve multiple public and private stakeholders, are transformational, and impact millions of people (Flyvbjerg 2014). Nowadays, rapid urbanization and globalization have led to the emergence of a growing number of megaprojects, such as high-speed rail lines, airports, seaports, and the Olympics. Due to the extreme complexity, the delivery of megaprojects requires many interconnected project constituents. One of the major challenges in successful delivery of megaprojects is to make all these project constituents work together as a whole effectively and efficiently.

A systems engineering approach has been adopted to deal with the challenge of integration in project systems in a number of recent studies. Systems integration is the process of making a system coherent by managing interactions across system elements (Whyte 2016). Existing studies have found systems integration as a promising approach

Post-doctoral Research Associate, Zachry Department of Civil Engineering, Texas A\&M University, College Station, US, jinzhu@civil.tamu.edu

2 Assistant Professor, Zachry Department of Civil Engineering, Texas A\&M University, College Station, US, amostafavi@civil.tamu.edu

3 Professor, Centre for Systems Engineering and Innovation, Imperial College London, London, UK, j.whyte@imperial.ac.uk 
for coping with complexity (Davies and Mackenzie 2014) and identifying risk and building resilience (Whyte 2016) in project systems.

Despite the growing literature on systems integration in complex projects, a formalized framework which can provide a taxonomy-based description of systems integration in megaprojects is still missing. Without a formalized framework, it is difficult to clearly identify, evaluate, and characterize systems integration at different levels of megaprojects. In addition, a standard basis for assessment of systems integration in different project systems may not be achievable. Thus, the objective of this paper is to propose such formalized framework towards systems integration theory in megaprojects using a system-of-systems (SoS) approach.

\section{MEGAPROJECTS AS SYSTEMS-OF-SYSTEMS}

Zhu and Mostafavi (2014) have identified that complex projects, such as megaprojects, are SoSs and thus should be investigated as SoSs. A SoS is an assemblage of components which individually may be regarded as operational and managerial independent systems (Maier 1998). In this study, the conceptualization of megaprojects as SoSs was adopted for the investigation of systems integration in megaprojects.

The conceptualization of megaprojects of SoSs in this study emphasizes the sociotechnical nature of megaprojects. Megaprojects consist of both social and technical elements. However, many of the existing conceptual structures of projects such as work breakdown structure (WBS) are solely based on the decomposition of physical outputs. This kind of conceptualizations of megaprojects have led to certain limitations. First, a hierarchical structure based on physical outputs is unique for each megaproject based on the functionality and design of that specific megaproject. Thus, an identical structure for analysis of systems integration at different levels of any megaproject is missing. Second, conceptualizations based on physical outputs usually ignore important social aspects (e.g., human decision making and information exchange) in megaprojects.

To this end, a SoS conceptualization which facilitates capturing both social and technical aspects at multiple levels of megaprojects was adopted is this study (DeLaurentis and Crossley 2005). Fig. 1 shows the megaproject SoS conceptualization structure. The five levels we identify in a megaproject as a socio-technical SoS are: SoS (megaproject), system (project), subsystem (process), activity, and base levels. At the SoS level, a megaproject SoS is a network of individual projects that are integrated towards a common goal of the megaproject. For example, a railway megaproject includes many individual projects (e.g., railway stations and sections). At the system level, each individual project, as an independent system, is a network of interconnected function processes (e.g., design, engineering, procurement, production/construction, marketing, project management, finance, safety management). The direct outcomes of these processes include physical components of the projects as well as management functions. At the subsystem level, each process, as a subsystem, is a network of different activities. Activities can be physical production, information processing, or decision making. For example, the design subsystem of a construction project includes activities such as obtaining design standards and codes, jobsite survey, communicating with owner regarding design requirements, and creating drawings. At the activity level, each activity is a network of interconnected baselevel entities (i.e., human agents, resources, and information). Each activity is accomplished by human agents utilizing certain information and resources. Finally, at the base level, human agent, resource, and information entities are abstracted and cannot be further decomposed. 
As shown in Fig.1, the hierarchical structure of megaproject SoSs leads to bi-directional integration across different levels. In a top-down perspective, the hierarchical structure is a deliverable-oriented decomposition of megaprojects. Systems integration can be realized by applying operability and functionality standards at the interfaces between decomposed components. In a bottom-up perspective, the hierarchical structure shows the aggregation from base-level entities to megaproject SoSs based on the dynamic interactions between different system elements.

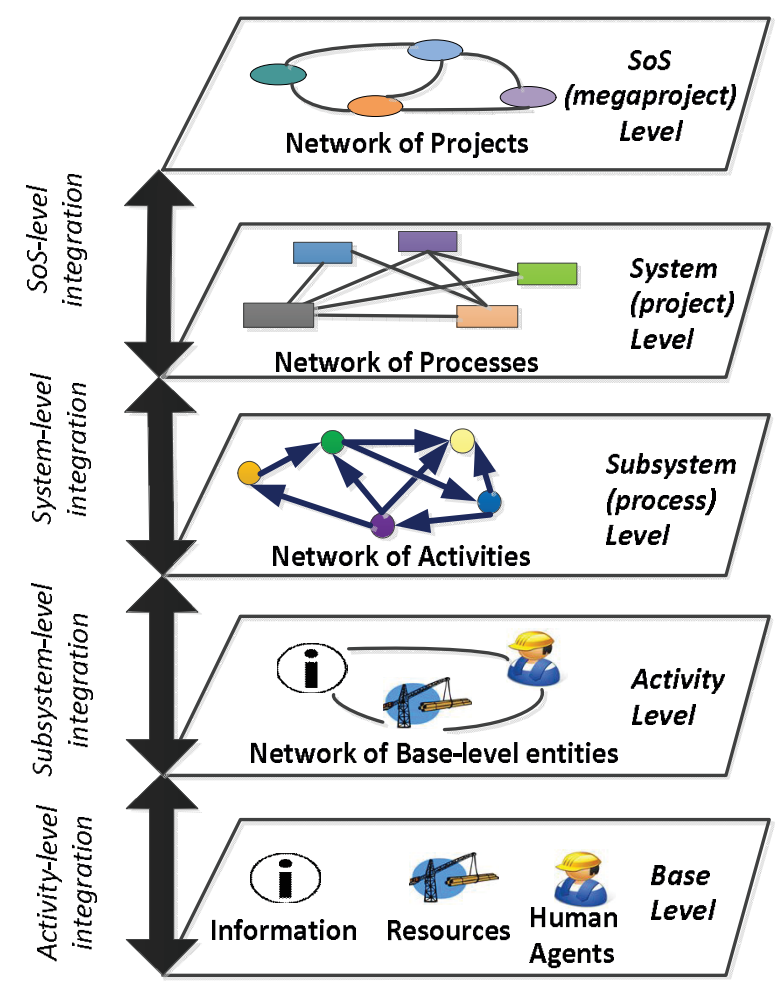

Figure 1: Megaproject system-of-systems conceptualization.

\section{A SOS FRAMEWORK TOWARDS SYSTEMS INTEGRATION THEORY}

Based on the SoS conceptualization of megaprojects, a SoS framework is proposed towards systems integration theory in megaprojects. Two major aspects of the proposed SoS framework are presented in this paper: dimensions and indicators of systems integration. These two aspects can provide a formalized platform for the description and assessment of systems integration and are the basis for further investigation of systems integration problems in megaprojects.

\subsection{Dimensions of Systems Integration}

Four dimensions of systems integration are identified in megaprojects: integration level, integration type, integration focus, and integration timing (Fig. 2).

First, systems integration in a megaproject occurs at four different levels: SoS level, system level, subsystem level, and activity level. At each of these levels, systems integration is the process of making the entities at the levels below working as a whole. For instance, SoS-level integration in a megaproject focuses on ensuring individual project systems with specific functions and objectives work coherently in order to achieve a 
common performance goal of the megaproject, while activity-level integration aims at aggregating base-level human agent, information, and resource entities effectively for the successful completion of activities. Systems integration at different levels can influence each other due to the bottom-up aggregation or top-down feedback mechanisms.
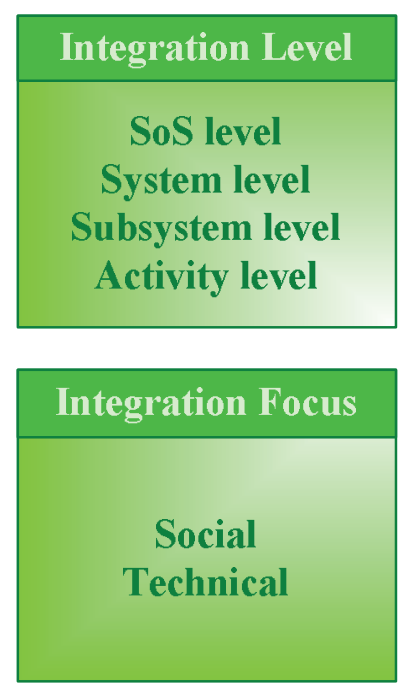

Figure 2: Dimensions of systems integration.
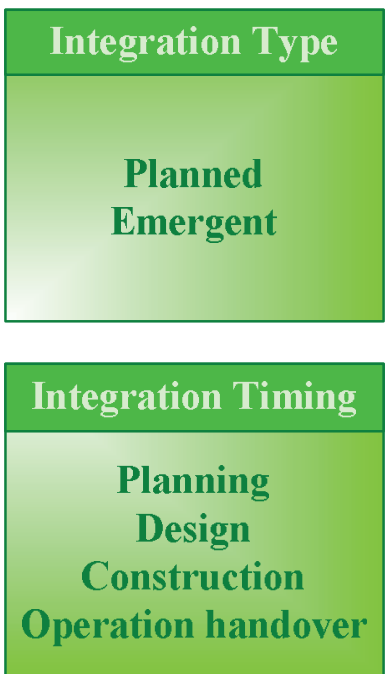

Second, there are two types of systems integration: planned integration and emergent integration (Bygstad et al. 2010). Planned integration is the process of applying standards at the interfaces between the decomposed components following a top-down approach (Whyte 2016). Planned integration is related to centralized design and control in megaproject management. In planned integration, the focus is on integrating individual projects at the SoS-level and different processes at the system-level. Hence, planned integration may not diffuse into subsystem and activity levels. Emergent integration, on the contrary, is the process of coordination and adaptation based on a bottom-up approach (Whyte 2016; Bygstad et al. 2010). Emergent integration can organically emerge at the activity and subsystem levels and result in integration at system and SoS levels. According to Bygstad et al. (2010), systems integration is partly planned and partly emergent.

The third dimension of systems integration in megaprojects is the focus of integration. As socio-technical systems, there are two focuses in the systems integration of megaprojects: social and technical (Bygstad et al. 2010). The technical integration focuses on the assembly of different technical artefacts, while the social integration focuses on human-centered interactions. The objective of technical integration is to address the technical challenges needed to transform inputs to outputs. The social integration is more concerned with the attributes of people (e.g., attitudes, skills, values), the relationships among people, reward systems, and authority structures (Bostrom and Heinen 1977). In megaprojects, emergent integration usually stems from social and organizational elements and ultimately permeates into technical elements. Hence, emergent integration can be investigated in couple with social integration.

The last dimension of systems integration is the timing of integration. Integration is a process instead of an outcome. Integration process takes place concurrently and repeatedly during each phase of a megaproject (Whyte 2016). Thus, systems integration can be assessed across four different phases of megaprojects: planning, design, construction, and operation handover. At different phases, the focuses and types of integration are different. 
For example, during the megaproject planning phase, systems integration is more focused on higher levels (i.e., system and SoS levels) and is usually planned integration. During the design and construction phases, when lower-level entities are more clearly defined and involved, emergent integration will occur more often. Finally, during the operation handover phase, the focus of systems integration returns to the higher levels of megaprojects in order to make sure there is no conflict between different project systems towards the common goal.

\subsection{Indicators of Integration}

Another important aspect in the proposed SoS framework is the degree of integration. According to Morris (1983), the degree or amount of integration describes how much "pulling together" the interfacing subsystems need in projects, and choosing the degree of integration across different interfaces requires considerable efforts. The degree of systems integration in a megaproject SoS affects the organizational structure and thus the functionality of the SoS. The optimal level of systems integration in a megaproject always depends on the scale, objective, complexity, phase, and dynamic environment of the project. Designing and controlling the degree of integration is an important management decision in megaprojects. In this study, seven indicators for measuring the degree of integration in complex systems (Kolasa 2005) were identified for the assessment of the degree of integration in the context of megaproject SoSs (Table 1).

Table 1: Indicators of integration in megaproject SoSs

\begin{tabular}{|c|c|}
\hline Indicators of integration & Application in megaprojects \\
\hline Coordination & $\begin{array}{l}\text { The condition where one component responds to the } \\
\text { action of another component at the same level }\end{array}$ \\
\hline $\begin{array}{l}\text { Exclusiveness of } \\
\text { interactions }\end{array}$ & $\begin{array}{l}\text { Each individual project system or subsystem has its own } \\
\text { execution procedure or communication protocols }\end{array}$ \\
\hline Interaction density & $\begin{array}{c}\text { The relative number of internal versus external } \\
\text { interactions }\end{array}$ \\
\hline Substitutability of parts & The speed and ease for SoS components to be replaced \\
\hline Proximity & Nearness in relationship, space, or time \\
\hline Autonomy of parts & $\begin{array}{c}\text { Freedom of components at different levels in independent } \\
\text { decision making and reaction }\end{array}$ \\
\hline Coherence & What happens in one part may also happen in other parts \\
\hline
\end{tabular}

(1) Coordination. Coordination is a condition where one component responds to the action of another component at the same level of a system (Kolasa 2005). In megaprojects, there are different types of coordination corresponding to different interfaces and interdependencies. There are three basic types of interdependencies in projects: pooled, sequential, and reciprocal (Davies and Mackenzie 2014). In pooled interdependency, each component provides a discrete contribution and may not directly interact with or respond to other components. Thus, coordination occurs when sequential and reciprocal interdependencies exist. In sequential interdependency, the output of one component is an input for another component, and, thus, there is a direct serial relationship. For example, in the construction process, the activities of installation of rebar and pouring concrete have 
a sequential relationship. For sequential interdependency, coordination mainly relies on planning and scheduling. In reciprocal interdependency, the dependency is mutual between two components and the outputs of each component become inputs for the other. For example, in a phased project, the interdependency between design and construction processes is reciprocal. For reciprocal interdependency, coordination requires information sharing and mutual adaptation. Thus, coordination corresponding to reciprocal interdependency is usually related to social aspects in megaprojects. In general, a greater level of coordination indicates a higher level of integration in megaproject systems. When using a network structure to model megaproject SoSs and links to capture different types of coordination, the number of links can be used to quantify the level of coordination.

(2) Exclusiveness of interactions. The exclusiveness of interactions indicates whether a component of a system maintains its individuality compared to other components at the same level (Kolasa 2015). In a megaproject, exclusiveness of interactions can be realized at the system and SoS levels based on the extent to which a number of managerially and operationally independent subsystems and systems are aligned while maintaining their independence. For example, the execution procedures and communication protocols in each individual project systems might be different with each other, which hinders the interactions between different project systems. Such exclusiveness of interactions may enhance the efficiency of a single system, however, it decreases the degree of integration at the SoS level.

(3) Interaction density. Interaction density is the number of internal versus external interactions. The internal interactions include the interactions within and across different levels of a megaproject. A megaproject also has interactions with external entities (e.g., government agencies, media, community) as a whole in order to facilitate the implementation of the megaproject. If a megaproject SoS is less integrated, the component systems or subsystems will need to interact with external entities by themselves. Thus, a greater interaction density (i.e., more internal interactions than external interactions) indicates a greater level of integration in a megaproject SoS.

(4) Substitutability of parts. Substitutability refers to the potential for system component to be effectively replaced by something else (Kolasa, 2005). In a megaproject, substitution of parts might be inevitable considering the extremely high levels of complexity and uncertainty. For example, due to a scope change, an individual project system might need to be substituted. Due to inefficiency at the system level, a subsystem might need to be completely or partially substituted (e.g., replace the project management team or change the procurement plan). The speed and ease of substitutability of parts may be different based on different organizational structures and cultures in megaprojects. For instance, in a traditional project setting, replacing a project director may have detrimental impacts on a megaproject. However, it might be easier to make such substitution in a networked or organic project organization. Exclusiveness of interactions in megaproject SoSs will hinder substitutability. In general, substitutability of parts decreases with systems integration.

(5) Proximity. In ecosystem science, proximity, as a required but not sufficient condition of integration, refers to proximity in space and time (Kolasa 2005). However, in megaproject SoSs, the proximity of different components is not limited to the constraint of space and time. Modern megaprojects are usually designed and constructed using advanced manufacturing techniques, and operated using digital control systems (Whyte 2016). Thus, different systems, subsystems, activities, and base-level entities can be integrated through advanced information and communication technologies even they are developed in different physical locations at different time. Thus, proximity, as an indicator 
of integration in megaproject SoSs, emphasizes more on the nearness of relationships and interdependencies (e.g., frequency of communication and coordination) other than space and time.

(6) Autonomy of parts. Autonomy of parts is an indicator of low integration. When the autonomy of parts is at its maximum, a system can be completely decomposed and no integrated system exists (Kolasa, 2005). Thus, in order to achieve a greater level of integration, a complete autonomy of parts should be prohibited. However, in megaproject SoSs, autonomy of components is not only inevitable but also necessary. For an individual project system or subsystem, certain degree of autonomy can avoid bureaucracy and facilitate quick response and reaction especially in emergent situations. For base-level human agents, their life experience in the Web 2.0 age has fostered their insistence on autonomy and participation in decision making when conducting activities (Levitt 2011), which provides opportunities for agility and innovation to emerge from the bottom up.

(7) Coherence. Coherence means that what happens in one part of a system propagates in all the other parts (Kolasa 2005). The existence of coherence shows the wholeness of a system and thus indicates a higher level of integration. The coherence in a megaproject can be assessed based on several phenomena, such as knowledge diffusion and shared culture and norms. For example, in a megaproject SoS, adoption of innovations (e.g., advanced information and communication technologies) in one project system may inspire the adoption of the same technology in other project systems, which shows the existence of coherence. Coherence is a systems integration indicator closely related to the social aspects in megaprojects.

The seven indicators provide a better understanding of systems integration in the context of megaproject SoSs. These indicators can be used to describe the level of integration, or for guiding towards a desirable level of integration in a megaproject SoS. There is no one best solution towards systems integration in megaproject SoSs. The tradeoffs between the impacts of different integration indicators need to be considered. For instance, one way to enhance systems integration is to eliminate exclusiveness of interactions by applying the same information processing tools and communication protocols in different systems and subsystems. It may increase the efficiency of coordination and interaction in megaprojects. However, it is costly and might increase the fragility to random disruptions (e.g., a failure of the shared information platform) (Ansar et al. 2016). Thus, decision makers in megaprojects need to develop project-specific management plans regarding system integrations based on the situation and priorities.

\section{CONCLUDING REMARKS}

In this paper, a SoS framework was proposed towards systems integration theory in megaprojects. The proposed framework was based on the conceptualization of megaprojects as project SoSs. Based on this conceptualization, different dimensions of systems integration and indicators of integration in megaproject SoSs were discussed.

The paper responds to the need for new taxonomies to characterise and evaluate integration in the context of megaprojects. It draws on and contributes to a growing literature on systems integration in megaprojects, bringing insights from other contexts in which integration is sought within complex systems to develop a formalized framework.

The proposed SoS framework provides a formalized platform for description and assessment of systems integration in megaprojects. Using the proposed SoS framework, various avenues of future work can investigate systems integration in different megaprojects under different uncertain environments, and ultimately lead to integrated 
theories pertaining to planning and management of systems integration in megaprojects. The future work directions include: (1) testing the proposed framework and propositions in this study using real-world case projects; and (2) exploring the impacts of different planning strategies (e.g., collaborative partnership, incentives for innovation) (Davies et al. 2009) on systems integration and identifying pathways leading to optimal level of systems integration in different scenarios.

\section{REFERENCES}

Ansar, A., Flyvbjerg, B., Budzier, A. and Lunn, D. (2016). Big is Fragile: An Attempt at Theorizing Scale, in: Flyvbjerg, B. (Ed.), The Oxford Handbook of Megaproject Management. Oxford University Press.

Bostrom, R.P. and Heinen, J.S. (1977). MIS Problems and Failures: A Socio-Technical Perspective, Part II: The Application of Socio-Technical Theory. MIS Quarterly, 1, 1128.

Bygstad, B., Nielsen, P.A. and Munkvold, B.E. (2010). Four Integration Patterns: A SocioTechnical Approach to Integration in IS Development Projects. Inf. Syst. J. 20, 53-80. doi:10.1111/j.1365-2575.2007.00280.x

Davies, A., Gann, D. and Douglas, T. (2009). Innovation in Megaprojects: Systems Integration at London Heathrow Terminal 5. Calif. Manage. Rev. 51, 101-125. doi:10.2307/41166482

Davies, A. and Mackenzie, I. (2014). Project Complexity and Systems Integration: Constructing the London 2012 Olympics and Paralympics Games. Int. J. Proj. Manag. 32, 773-790. doi:10.1016/j.ijproman.2013.10.004

DeLaurentis, D. A. and Crossley, W. A. (2005). A Taxonomy-based Perspective for System of Systems Design Method, in: IEEE International Conference on Systems, Man, and Cybernetics. pp. 86-91.

Flyvbjerg, B. (2014). What You Should Know about Megaprojects and Why: An Overview. Proj. Manag. J. 45, 6-19. doi:10.1002/pmj

Kolasa, J. (2005). Complexity, System Integration, and Susceptibility to Change: Biodiversity Connection. Ecol. Complex. 2, 431-442. doi:10.1016/j.ecocom.2005.05.002

Levitt, R.E. (2011). Towards Project Management 2.0. Eng. Proj. Organ. J. 1, 197-210. doi:10.1080/21573727.2011.609558

Maier, M.W. (1998). Architecting Principles for Systems-of-systems. Syst. Eng. 1, 267-284.

Morris, P.W.G. (1983). Managing Project Interfaces: Key Points for Project Success, in: Project Management Handbook. p. 16-55. doi:10.1002/9780470172353

Whyte, J. (2016). The Future of Systems Integration within Civil Infrastructure: A Review and Directions for Research, in: INCOSE International Symposium. Edinburgh, Scotland, pp. 1541-1555.

Zhu, J. and Mostafavi, A. (2014). Towards A New Paradigm for Management of Complex Engineering Projects: A System-of-systems Framework, in: 2014 IEEE International Systems Conference Proceedings. pp. 213-219. doi:10.1109/SysCon.2014.6819260. 